Research Article

\title{
EDF-Adaptive: A New Semipartitioned Scheduling Algorithm for Multiprocessor Real-Time
}

\author{
Shujuan Huang $\mathbb{D},{ }^{1}$ Tiansen Li $\mathbb{D},{ }^{1}$ Zhihao Ma $\mathbb{D}^{1},{ }^{1}$ Feng Xiao $\mathbb{D}^{1},{ }^{1}$ and Wenjuan Zhang $\mathbb{D}^{2}$ \\ ${ }^{1}$ School of Computer Science and Engineering, Xi'an Technological University, Xi'an 710021, China \\ ${ }^{2}$ School of Sciences, Xi'an Technological University, Xi'an 710021, China
}

Correspondence should be addressed to Shujuan Huang; huangshujuan@xatu.edu.cn

Received 18 October 2021; Revised 10 November 2021; Accepted 11 November 2021; Published 13 December 2021

Academic Editor: Gengxin Sun

Copyright (c) 2021 Shujuan Huang et al. This is an open access article distributed under the Creative Commons Attribution License, which permits unrestricted use, distribution, and reproduction in any medium, provided the original work is properly cited.

\begin{abstract}
Most of the multiprocessor real-time scheduling algorithms follow the partitioned approach, the global approach, or the semipartitioned approach which is a hybrid of the first two by allowing a small subset of tasks to migrate. EDF-fm (Earliest Deadline First-based Fixed and Migrating) and EDF-os (Earliest Deadline First-based Optimal Semipartitioned) are semipartitioned approaches and were proposed for soft real-time sporadic task systems. Despite their desirable property that migrations are boundary-limited such as they can only occur at job boundaries, EDF-fm and EDF-os are not always optimal and have higher tardiness and cost of overheads due to task migration. To address these issues, in this paper, we classify the systems into different types according to the utilization of their tasks and propose a new semipartitioned scheduling algorithm, earliest deadline first-adaptive, dubbed as EDF-adaptive. Our experiments show that EDF-adaptive can achieve better performance than EDF-fm and EDF-os, in terms of system utilization and tardiness overhead. It is also proved that EDFadaptive is able to lessen the task migration overhead, by reducing the number of migrating jobs and the number of processors to which a task is migrated.
\end{abstract}

\section{Introduction}

The partitioned and global approaches are the classical scheduling algorithms for real-time multiprocessor systems. Because the former assigns tasks statically to processors and does not allow the tasks to migrate, it cannot achieve higher system utilization whereas the latter schedules tasks from a single run queue and the task hence can be migrated [1]. Although the global approach can attain higher system utilization but migrating tasks entails higher run-time overheads, especially in soft real-time (SRT) systems, many approaches have been proposed, based on these two algorithms [2-6] or the hybrid of the two [7-18]. Some approaches are aimed at reducing the switch context or preemption overheads and achieving higher system utilization [19-22].

Semipartitioned scheduling extends the partitioned scheduling by allowing a (usually small) subset of tasks to migrate, and these tasks cannot be feasibly assigned to two or more processors by a partitioned scheduling algorithm [23-26]. This type of approach is different from the global ones as the former use push migrations which are planned before execution whereas the latter use pull migrations which are reactive in nature and more difficult to account for and implement efficiently.

In this paper, we are particularly interested in two semipartitioned scheduling algorithms, EDF-fm [12] and EDF-os [13], because they are adaptive to SRT systems and allow a migrating task to migrate only between job boundaries, i.e., successive invocations. However, EDF-fm deals with pertask utilization restrictions according to the releasing sequence and cannot fully utilize the underlying hardware platform's available capacity that makes it nonoptimal. These restrictions preclude any task utilization from exceeding half the capacity of a processor. EDF-fm has two phases as follows: 
(1) Offline assignment, where the tasks are assigned to the processors and meanwhile the tasks' attributions of fixed or migrating are given

(2) Online execution, where the extended earliestdeadline-first (EDF) scheduling is used to execute fixed tasks and migrating tasks on each processor. Note that a fixed task, i.e., a nonmigrating task, is the task assigned to only one processor and a migrating task is the task assigned to two or more processors in the phase of offline assignment

Like EDF-fm, EDF-os also has two phases, and in the online execution phase, the migrating tasks are statically prioritized over fixed ones. This ensures migrating tasks never miss deadlines. However, in the assignment phase, EDF-os is very different from EDF-fm. It assigns tasks as fixed ones as many as possible using a worst decreasing bin-packing heuristic. All remanent tasks are assigned in decreasing utilization order, by considering each processor and remanent tasks in turn. This approach allows migrating tasks to execute on any number of processors, instead of just two. However, the preemption overheads will be increased.

Though EDF-os increases the number of fixed tasks and reduces the number of migrating tasks, it may schedule a migrating task on three or more processors and thus the actual migrating times do not decrease. As a result, since the context switching overheads are also determined by the number of migrating jobs and the preemption times, EDFos still has higher context switch overheads.

In this paper, we propose a novel EDF-based approach that overcomes the limitations of EDF-fm and EDF-os by reducing the number of jobs that miss deadline and making a balance between the cost of overheads and system utilization. The approach partitions tasks and schedules them adaptively, according to their utilization; thus, we dub it as EDF-adaptive. Given a real-time multiprocessor system with periodic tasks, EDF-adaptive first divides the tasks into two sets: one with high utilization denoted as $S^{H}$ and the other with low utilization denoted as $S^{L}$. EDF-adaptive then chooses tasks from $S^{H}$ and $S^{L}$ whose utilization sum to an integer is less than the number of processors. We call these tasks complimentary. This selection process is repeated until the sum of all integers equals to the number of processors. During the execution, EDF-adaptive separates the processors into different scheduling queues according to the integers and puts these tasks into the corresponding queue. Our experiments have demonstrated that, compared with EDF-fm and EDF-os, EDF-adaptive is able to achieve lower job switching overheads, and the lowest degree of splitting and migrating, while in the meantime maintaining competitiveness in terms of system utilization and slack task minimum tardiness.

The remainder of this paper is organized as follows. Related works of semipartitioned algorithms are described in Section 2. The system model, definitions, and theorems about different utilization systems and EDF-adaptive algorithm in detail are introduced in Section 3. Experimental evaluations are undertaken to demonstrate the effectiveness of the proposed algorithm in Section 4. Finally, concluding remarks are drawn in Section 5.

\section{Related Work}

As mentioned in the previous section, there are two typical categories of scheduling algorithms for a real-time multiprocessor system, i.e., the global and the partitioned. Since our proposed approach in this paper concerns more on the latter, here, we focus on our review on semipartitioned schedulers, both for hard real-time (HRT) and soft real-time (SRT) systems. The difference between them is that the tasks in HRT are not allowed to miss their deadlines whereas those in SRT are allowed.

A variety of semipartitioned schedulers have been proposed for HRT that does not consider job boundarylimited. EDF-WM (Earliest Deadline First with Window constraint Migration) [14] assigns most tasks to processors, allowing some tasks to migrate between processors. But it is not only non-boundary-limited but also works by splitting tasks in a way guaranteeing no job misses a deadline while scheduling all jobs. When all processors share a single run queue, G-FL (Global Fair Lateness) [15] provides lateness bound for all tasks. For each job, G-FL uses a PP (Priority Point) that precedes its deadline. G-FL schedules each job based on the max-lateness bound which is minimized for any task system. C-FL (Clustered Fair Lateness) [16] defines the split factor of a task as the number of subjobs into which each of its jobs is split. By splitting job budgets to create subjobs with shorter periods and the worst-case execution times, such bounds can be reduced to near zero for implicit deadline sporadic task systems. Proposed for periodic task systems, EKG (Earliest Deadline First with task splitting and $\mathrm{K}$ processors in a Group) [17] is HRT-optimal when a configurable parameter is reduced in a way that increases preemption frequency.

Because non-boundary-limited schedulers above mentioned allow jobs to migrate in any time, it will be expensive in practice if jobs maintain a much-cached state. Some semipartitioned algorithms have been proposed to support SRT systems under bounded deadline tardiness. EDF-fm was first proposed, requiring utilization constraints that render it nonoptimal. To overcome the constraints, EDF-os was proposed by scheduling migrating tasks on three or more processors. Since none of the other migration algorithms mentioned above are job-boundary-limited, we will compare our proposed approach with EDF-fm and EDF-os.

\section{Task Model and Scheduling Algorithm}

3.1. Task Model. Assume a sporadic system consists of $M$ processors and owns $N>M$ tasks $\tau=\left\{\tau_{1}, \tau_{2}, \cdots \tau_{N}\right\}$. These sporadic tasks are independent and to be scheduled upon a multiprocessor platform with $M \geq 2$ identical processors. In a sporadic task model, a sporadic task is a four-tuple $\tau_{i}\left(r_{i}, e_{i}, d_{i}, p_{i}\right)$ where $e_{i}, d_{i}$, and $p_{i}$ are positive integer numbers and $r_{i}$ is a nonnegative integer number and it 
is characterized by a minimum interarrival time, also referred to as its period $p_{i}$, a worst-case execution cost $e_{i}$ $p_{i}$, and a relative deadline $d_{i}$ and a release time $r_{i}$. Every task $\tau_{i}$ may be invoked zero or more times with two consecutive invocations separated by at least $p_{i}$, time units. Each invocation of $\tau_{i}$ is referred to as a job of $\tau_{i}$. The $k^{\text {th }}$ job of $\tau_{i}$, where $k \geq 1$, is denoted as $J_{i, k}$. The release time of the job $J_{i, k}$ is denoted as $r_{i, k}$. Each job of $\tau_{i}$ is executed for at most $e_{i}$, time units. The absolute deadline (or simply, deadline) of $J_{i, k}$, denoted as $d_{i, k}$, is the time at or before which $J_{i, k}$ should complete execution. Each task is sequential and at any time may be executed on at most one processor.

A periodic task system, in which every two consecutive jobs of every task $\tau_{i}$ are separated by $p_{i}$ time units, is a special case of a sporadic task system. In this paper, we focus on the periodic task system and assume that $d_{i}=p_{i}$ holds for all $\tau_{i}$ and the first job of each task will be invoked or released at time zero. A task can then be represented using a two-tuple $\tau_{i}\left(e_{i}, d_{i}\right)$. The $t^{\text {th }}$ processor is denoted as $P_{l}$, where $1 \leq l \leq M$. All tasks are synchronous and periodic and have the same initial release date. They are preemptive and independent. The overheads of other shared resources between tasks are not considered.

3.1.1. Utilization System and Tasks. To facilitate the description of our proposed EDF-adaptive, we categorize the real-time system aforementioned according to its task utilization.

Define $u_{i}=e_{i} / d_{i}$, where $u_{i}$ is the utilization of task $\tau_{i}$. Then, we have the utilization for all tasks $U(\tau)=\sum_{\tau_{i} \in \tau} u_{i}$.

We define the real-time system as follows:

(i) Single full utilization system, if $U(\tau)=1$

(ii) All full utilization system, if $U(\tau)=M$

(iii) $K$ full utilization system, if $U(\tau)=K$, where $K$ is an integer. Under this scenario, the system may be either underloaded if $M>K>1$ or fully utilized if $K=M$. For the latter, it may be an all full utilization system $K=M \geq 2$ or a single full system $K=M=1$; overloaded utilization system, if $U(\tau)>M$

For the overloaded utilization system, we schedule the largest subset $\tau_{\text {sub }}$ so that the total utilization of the tasks' subset is very close to $M$. That is, $M-\sum_{i=1}^{k} u_{i}$ where $\varepsilon \in(0$, 1 ) is a minimum float number and $k$ is the number of the tasks in the subset $\tau_{\text {sub }}$.

Each task is allocated a nonzero fraction, or share of one processor with the available utilization of 1.0. If there are $k$ tasks being scheduled on the $j^{\text {th }}$ processor, the remanent utilization of this processor is

$$
U_{j}^{\tau}=1-\sum_{i=1}^{k} u_{i}
$$

where the fraction of the $\tau_{i}$ 's utilization scheduled on this processor $p_{j}$, denoted as $u_{i, j}$, is $U_{j}^{\tau}$. The total remanent utilization of this system is

$$
U_{\mathrm{rem}}^{\tau}=M-\sum_{j=1}^{M} U_{j}^{\tau}
$$

We define $\tau_{i}$ as a high utilization task if $u_{i} \geq 1 / 2$, and a low utilization task otherwise. Define $S^{H}$ as a high utilization task set consisting of high utilization tasks, and $S^{L}$ as a low utilization task set consisting of low utilization tasks. We represent $S^{H}$ and $S^{L}$ as follows:

$$
\begin{aligned}
& S^{H}=\left\{\tau_{i} \mid u_{i} \geq \frac{1}{2}\right\}, \\
& S^{L}=\left\{\tau_{i} \mid u_{i}<\frac{1}{2}\right\} .
\end{aligned}
$$

A high utilization system is defined as a system consisting of only $S^{H}$ set.

3.1.2. Theorems for EDF-Adaptive Utilization. Here, we define several new theorems for the EDF-adaptive algorithm. First, we propose a concept of complementary tasks.

Definition 1. Given $k$ periodic tasks $\tau_{1}, \tau_{2}, \cdots \tau_{k}, k>1$, we regard $k$ tasks are complementary if $\sum_{i=1}^{k} u_{i}=1$. Then, we have the following lemma.

Lemma 2. Complementary periodic tasks can be scheduled on uniprocessor, and their total utilization is 1.

According to the DD scheduling algorithm [18], this lemma can be easily proved.

Given a time interval $\Delta$, CPU utilization $U(\Delta)=\sum_{i=1}^{k} u_{i}$, where $k$ is a set of periodic tasks executed in $\Delta$, and $u_{i}$ is the ratio of the execution time of $\tau_{i}$ task to $\Delta$. Also, define the remanent utilization rate of a processor as $\beta(\Delta)=1-$ $U(\Delta)$; then, the remaining time unit is

$$
\operatorname{spare}(\Delta)=\lfloor\beta(\Delta) \times \Delta\rfloor=\lfloor(1-U(\Delta)) \times \Delta\rfloor .
$$

Let $T$ be the minimum common multiple of $n$ tasks' period. Given $\sum_{i=1}^{n} u_{i} \leq 1$ if $n$ tasks can be scheduled in $[0$, $T$ ), then $n$ tasks can be scheduled for the entire period of execution, according to [18]. This is because the corresponding scheduling method can be executed repeatedly in the future $[i * T,(i+1) * T]$, where $i \geq 1$.

Lemma 3. Given $n$ preemptive periodic tasks allocated to the $j^{\text {th }}$ processor, the available time unit of the $i^{\text {th }}$ periodic task $\tau_{i}=\left(e_{i}, d_{i}\right)$ in $[0, T]$ is spare $=T \times\left(1-\sum_{k=1}^{n} u_{k}\right)$, where $T$ is the minimum common multiple of the period of $n$ tasks and $\tau_{i}$. Define $x$ as the available average time unit allocated to each activation of $\tau_{i}$, and $l$ as the number of $\tau_{k}$ 'jobs allocated to this processor. 
We have

(1) A necessary and sufficient condition for $\tau_{i}$ to be assigned to the $j^{\text {th }}$ processor as a fixed task without tardiness in $[0, T]$ is $x \geq e_{i}$ where $l=T / d_{i}$ and $x=$ spare/l

(2) If $\tau_{i}$ is to be assigned to the $j^{\text {th }}$ processor as a migrating task and $u_{i, j}$ is the fraction of the $\tau_{i}$ 'utilization is assigned to $P_{j}$, then $l=\left\lceil\left(T / d_{i}\right) \times\left(u_{i, j} / u_{i}\right)\right\rceil$ and $x=$ spare/l. A sufficient condition for $\tau_{i}$ to be assigned to the $j^{\text {th }}$ processor as a migrating task without tardiness in $[0, T]$ is $x \geq e_{i}$

Proof. For fixed tasks, firstly, we prove the necessary conditions under two scenarios.

One is for the first scheduled task, i.e., $n=1$ and $\tau_{1}\left(e_{1}\right.$, $d_{1}$ ), where $e_{1}$ is the execution time and $d_{1}$ is the deadline or period. Obviously, this task is assigned to the processor as the fixed task. Given that the current spare time unit spare $=T=d_{1}$ time units and only $\tau_{1}$ is allocated to this processor, we get

$$
x=\frac{\text { spare }}{T / d_{1}}=d_{1} \geq e_{1}
$$

The other is when $n=k-1$, and the remanent time unit is spare $=T \times\left(1-\sum_{i=1}^{k-1} u_{i}\right)$, we need to decide if the $k^{\text {th }}$ task $\tau_{k}\left(e_{k}, d_{k}\right)$ will be allocated to this processor as a fixed task. $T$ time unit including $\tau_{k}$ tasks' activation times is $l=T / d_{k}$. Because the time units that must be executed in each activation time are $e_{k}$ and $\tau_{k}$ are scheduling on this processor with no tardiness, the remanent time unit spare must not be less than $l \times e_{k}$. Then, we get $x=$ spare $/ l \geq e_{k}$.

Secondly, we prove sufficient conditions. When $n=k-1$ , the remanent time unit is spare $=T \times\left(1-\sum_{i=1}^{k-1} u_{i}\right)$. Because $T$ is the minimum common multiple of the period of $n$ tasks and $\tau_{i}, T$ is dividable to $d_{k}$. If the $k^{\text {th }}$ task $\tau_{k}\left(e_{k}, d_{k}\right)$ is assigned to this processor as a fixed task, the activation times of $\tau_{k}$, in $[0 ; T]$, is $l=T / d_{k}$ and the available average time unit allocated to each activation is $x=$ spare $/ l$. The time units that must be executed in each period are $e_{k}$. If $x \geq e_{k}$, there are enough time units for $\tau_{k}$ 's every activation with no tardiness.

For migrating tasks, when $n=k-1$, the remanent time unit is spare $=T \times\left(1-\sum_{i=1}^{k-1} u_{i}\right)$; we need to decide if the $k^{\text {th }}$ task $\tau_{k}\left(e_{k}, d_{k}\right)$ will be allocated to this processor as a migrating task. Let $u_{k, j}$ be the fraction of $\tau_{k}$ 's utilization. We can obtain the proportion of jobs allocated to this processor by $u_{k, j} / u_{k}$. The total activation time of $\tau_{k}$ in $[0, T]$ is $T / d_{k}$, but there are at most $\left\lceil\left(T / d_{k}\right) \times\left(u_{k, j} / u_{k}\right)\right\rceil$ jobs of $\tau_{k}$ allocated to this processor in $[0, T]$. Let $l$ be the number of jobs, $l=\left\lceil\left(T / d_{k}\right) \times\left(u_{k, j} / u_{k}\right)\right\rceil$. If $x=$ spare $/ l \geq e_{k}$, there are enough time units for $\tau_{k}$ 's jobs to be scheduled on this processor without any tardiness.
According to Lemma 3, given a task $\tau_{i}$ for a processor, we can decide whether it should be regarded as a fixed task or migrating task. If $n-1$ tasks are assigned as fixed task and for the $n^{\text {th }}$ task there always exists $x<e_{i}$ in $[0, T], \tau_{n}$ is then removed. If the system becomes an underloaded utilization system after the removal, we call $\tau_{n}$ is a probable slack task.

Theorem 4. A probable slack task $\tau_{i}\left(e_{i}, d_{i}\right)$ can be delayed, and its minimum tardiness is

$$
\text { Tardiness }_{\min }=e_{i} \times\left(\frac{1}{M-\sum_{i \neq j} u_{j}}-\frac{1}{u_{j}}\right) \text {. }
$$

Proof. If a probable slack task $\tau_{i}$ is removed, the system's remanent utilization is $M-\sum_{i \neq j} u_{j}>0$. To schedule this system, the task has to be delayed in the whole process, and we assume the minimum period after delay is $d_{\text {new }}$, which is also the delay deadline for the soft real-time task. The tardiness can be derived as

$$
\begin{aligned}
\text { Tardiness }_{\text {min }} & =d_{\text {new }}-d_{i}=e_{i} \times\left(\frac{1}{M-\sum_{i \neq j} u_{j}}\right)-d_{i} \\
& =e_{i} \times\left(\frac{1}{M-\sum_{i \neq j} u_{j}}-\frac{1}{u_{i}}\right) .
\end{aligned}
$$

3.2. EDF-Adaptive Algorithm. Similar to EDF-fm and EDFos, our EDF-adaptive also has two phases: offline task partitioning phase and task splitting execution phase.

3.2.1. Task Partitioning Algorithm. To partition tasks, we first arrange them in utilization ascending order. From $S^{H}$ and $S^{L}$, we find all complementary task sets with their total utilization being $K(1 \leq K \leq M)$. Subsequently, we dispatch $K$ sets into the $K$ processors' scheduling queue. We assume that a center processor distributes new tasks to processors, and each processor sends a request and amends its own scheduling queue.

Though the DD scheduling algorithm can be used for the tasks in one processor with its scheduling queue, there exist two issues. One is that the complementary task sets may not be unique. For example, given six tasks with utilization as $\{0.1,0.3,0.4,0.5,0.7,0.9\}$, we can divide them into two optional complementary tasks set: one is $\{0.1,0.4,0.5\}$, $\{0.7,0.3\}$ and the other is $\{0.1,0.9\},\{0.7,0.3\}$. EDF-adaptive always chooses the sets that include most tasks, unless the system species the priority of tasks. The other issue is to select the parameter $\varepsilon$ so that all similar complementary tasks can be obtained. EDF-adaptive selects $\varepsilon$ so that the total utilization is the nearest close to $K$.

Algorithm 1 presents the pseudocode to find complementary tasks set for $K$ full utilization system. As shown in Algorithm 1, the overall approach is to find and add complementary tasks into Set $_{\text {comp }}$ and then remove them from $Q$. 


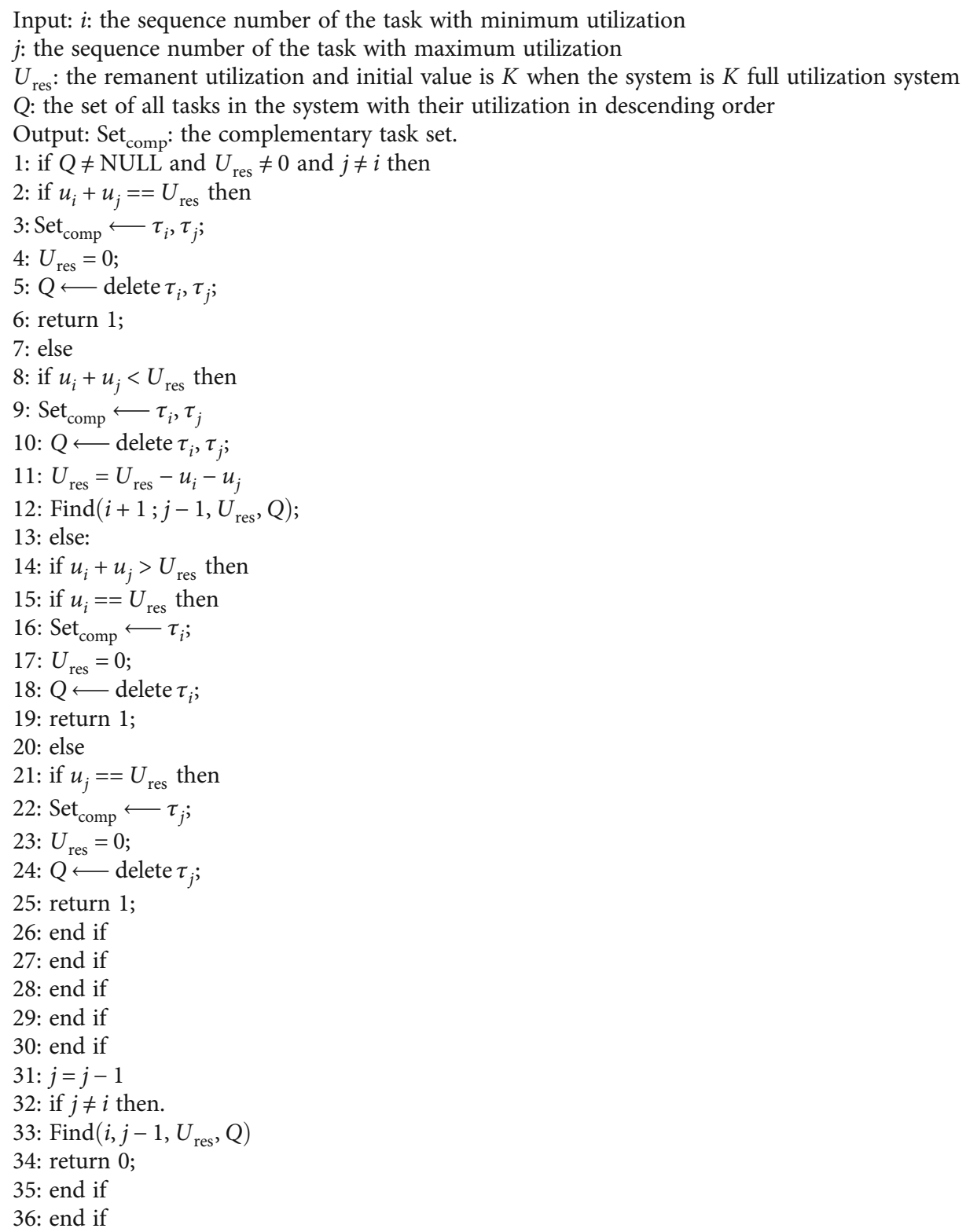

Algorithm 1: Find complementary tasks.

When there are still tasks in $Q$ or $U_{\text {res }}$ is not 0 or $j=i$, Algorithm 1 recursively finds the complementary tasks set by adding $u_{i}$ and $u_{j}$ that is depending on the relationship between $u_{i}, u_{j}$, and $U_{\text {res }}$; there are several cases as follows: set.

If $u_{i}+u_{j}==U_{\text {res }}, \tau_{i}$ and $\tau_{j}$ belong to the complementary

If $u_{i}+u_{j}>U_{\text {res }}$, only one of them can be an element in the complementary set, depending on if $u_{i}==U_{\text {res }}$ or $u_{j}=$ $=U_{\text {res }}$.

If $u_{i}+u_{j}<U_{\text {res }}, \tau_{i}$ and $\tau_{j}$ may or may not be the elements in the complementary set.

If none of the conditions above is satisfied, the algorithm updates the sequence number $(j=j-1)$ and continues to search.
3.2.2. Task Splitting and Execution Algorithm. To achieve higher system utilization, we split tasks assigned to $K$ processors according to their utilization.

First, the tasks are arranged in descending order of utilization in the queue. The tasks with the highest utilization are assigned to the $j^{\text {th }}$ processors and then removed from the queue. If $j \leq K$, calculate the remanent utilization of the $j^{\text {th }}$ processor $U_{j}$. If $U_{j}>0$, select the task which has the lowest utilization in the queue and calculate the split value by

$$
f_{i, j}=\left(\frac{T}{d_{i}}\right) \times\left(\frac{u_{i, j}}{u_{i}}\right)
$$


Algorithm 2: Task splitting.

where $f_{i, j}$ is the number of jobs that $\tau_{i}$ will be scheduled on the $j^{\text {th }}$ processor in $[0, T]$, according to Lemma 3 . The task $\tau_{i}$ will be allocated to execute on the $j^{\text {th }}$ processor in time $[0, T]$, i.e., the units of execution time units of $\tau_{i}$ on the $j^{\text {th }}$ processor. $\tau_{i}$ will be divided and assigned to other processors if its remanent execution time unit is not zero. If $U_{j} \leq 0$, it means that the $j^{\text {th }}$ processor has completed the assignment; if $j>K$, it indicates that the tasks of this group set have been allocated over, and it returns the corresponding processor with the allocated tasks. The pseudocode of the splitting approach is presented in Algorithm 2.

As shown in Algorithm 2, there are the following two scenarios:

If $U_{i} \geq U_{j}$, it means $U_{j}$ cannot satisfy $U_{i}$. The $\tau_{i}$ 's utilization share on processor $P_{j}$ is $u_{i, j}=U_{j}$, and the remaining utilization of $\tau_{i}$ is $U_{i}=U_{i}-U_{j}$. The number of jobs that $\tau_{i}$ will be scheduled on the $j^{\text {th }}$ processor in $[0, \mathrm{~T}]$ is $f_{i, j}$.

If $U_{i}<U_{j}$, it means that $U_{j}$ can satisfy $U_{i}$. The share of task $\tau_{i}$ assigned on processor $P_{j}$ is $u_{i, j}=U_{j}$. We calculate the number of jobs $\left(f_{i, j}\right)$ that $\tau_{i}$ will be scheduled on the $j^{\text {th }}$ processor in $[0, \mathrm{~T}]$ and record $f_{i, j}$ in the scheduling queue of processor $P_{j}$. Next, we update the remaining utilization of $P_{j}, U_{j}=U_{j}-U_{i}$, and remove the task $\tau_{i}$ from the complementary set before selecting the next task. If $U_{j}=0$, it means that the remaining utilization of the $j^{\text {th }}$ processor has been exhausted and the next processor must be selected.

In the execution phase, all released jobs of the fixed tasks are scheduled online without migration. Different from EDF-os and EDF-fm that the priority of migration tasks is higher than that of fixed tasks at any time, in EDFadaptive's execution phase; the priority of migration tasks is only higher than that of fixed tasks with lower utilization. To compare the task splitting and scheduling of EDFadaptive with EDF-fm and EDF-os, we use a task system $\tau=\{(9,10),(7,12),(3,4),(2,3),(3,5),(1,2)\}$ as an example, since $U(\tau)=4$ is feasible on four processors. Because all task utilization but one exceed 0.5 and the other utilization is exactly 0.5 , all the tasks belong to $S^{H}$. The comparing results are shown in Figures 1-6.

Figure 1 illustrates the offline assignment phase of EDFfm functions, where tasks are assigned to the processors as either fixed task or migrating one. We can see that EDF- 


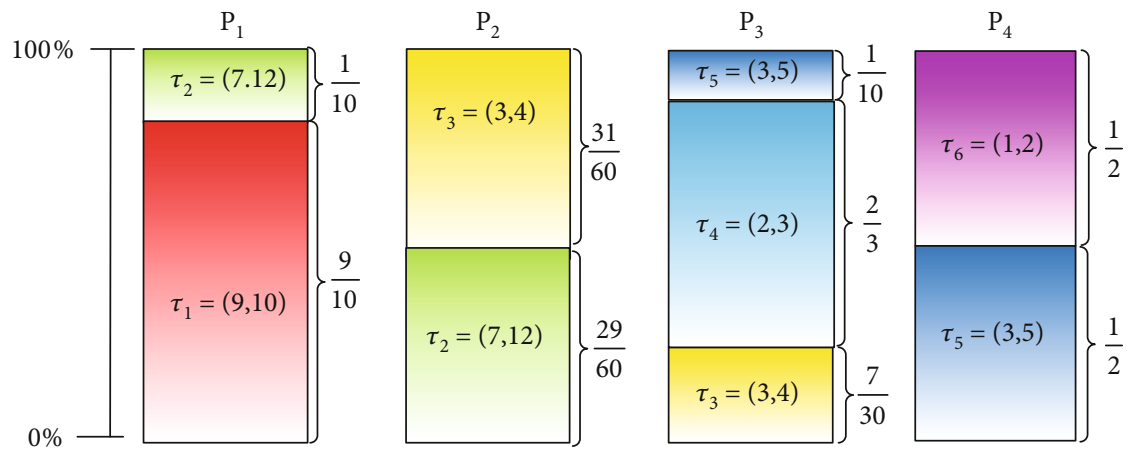

FIGURE 1: EDF-fm offline assigning tasks in 4 processors.

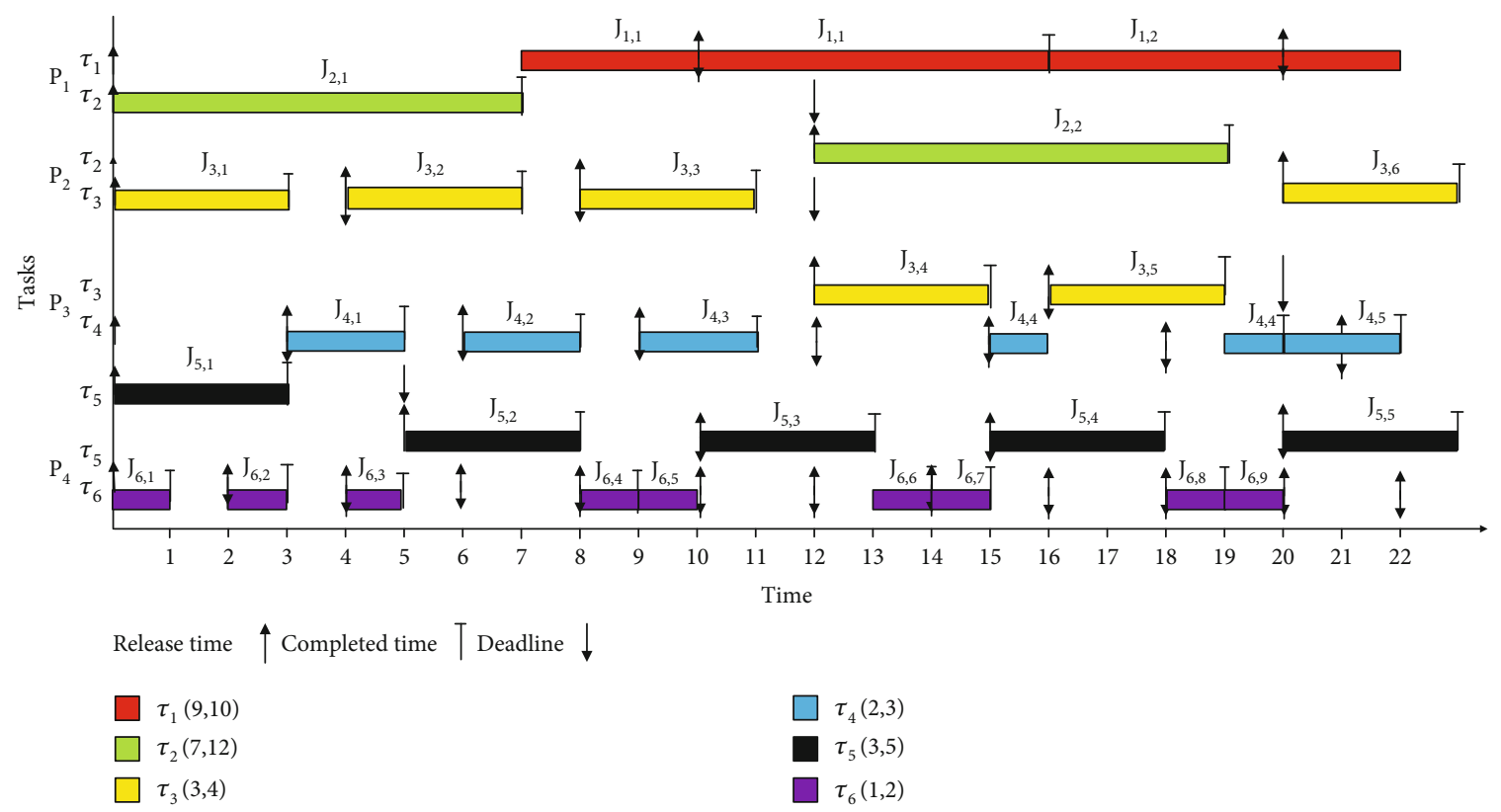

FIgURE 2: EDF-fm online scheduling results in 22 time units.

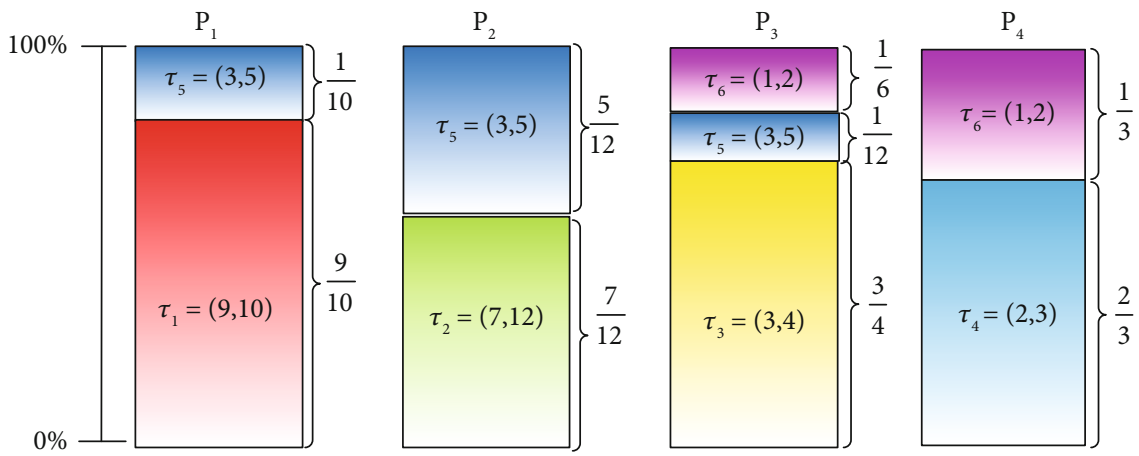

FIgURE 3: EDF-os offline assigning tasks in 4 processors.

fm has a simple assignment algorithm which dispatches tasks according to their order of coming. If the currently considered processor $P_{j}$ has sufficient unallocated utilization, the currently considered task $\tau_{i}$ is assigned to it as a fixed task and $u_{i, j}=U_{i}$; otherwise, $\tau_{i}$ exhausts the remaining unallocated utilization of $P_{j}$ and receives from $P_{j+1}$ the rest of its allocation required. For example, $\tau_{2}$ is assigned to $P_{1}$ and $P_{2}$, and its utilization has been divided into two parts: $u_{2,1}=1 / 10$ and $u_{2,2}=u_{2}-u_{2,1}=29 / 60$; that means, $\tau_{2}$ is a migrating task. 


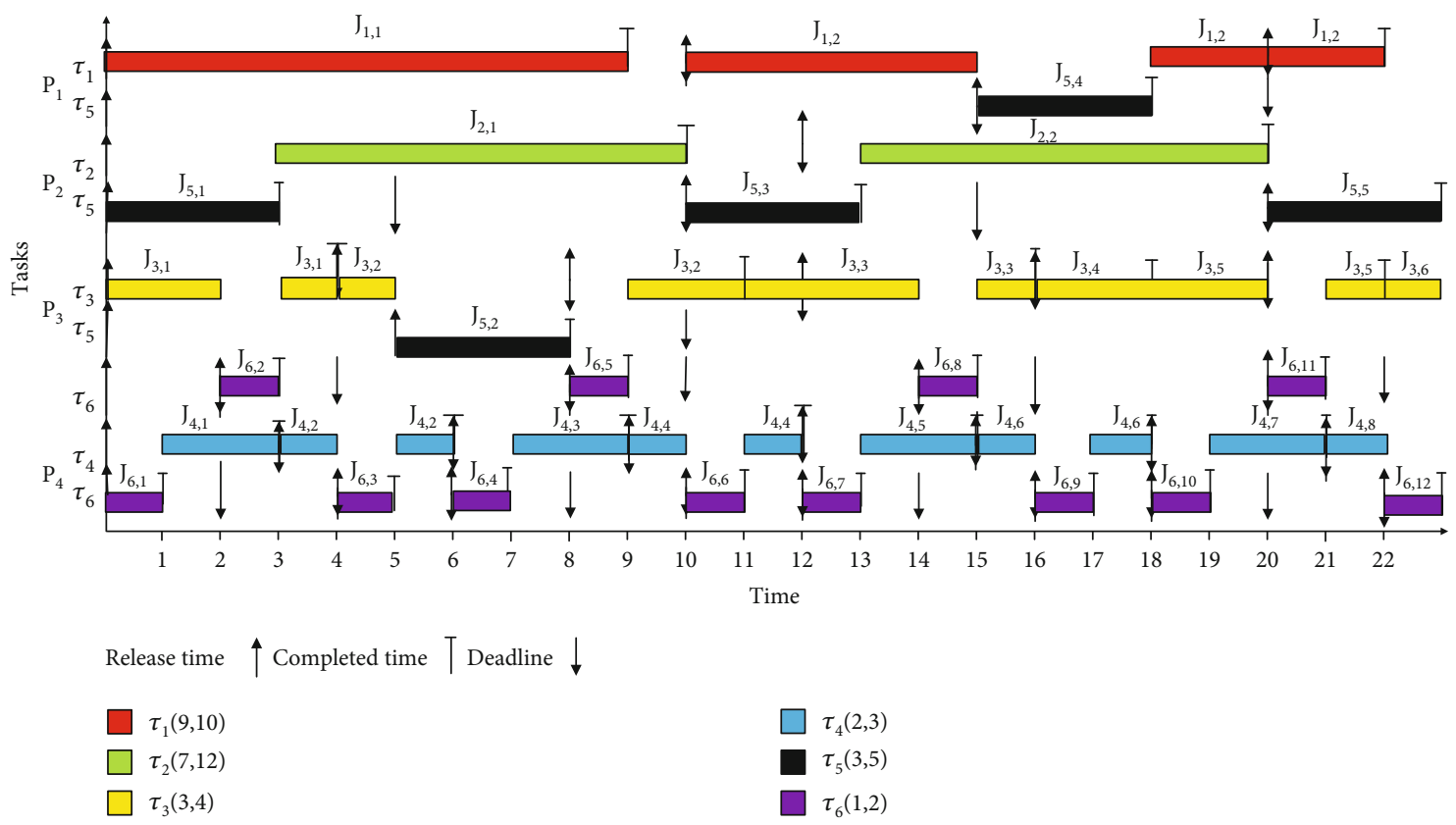

Figure 4: EDF-os online scheduling results in 22 time units.

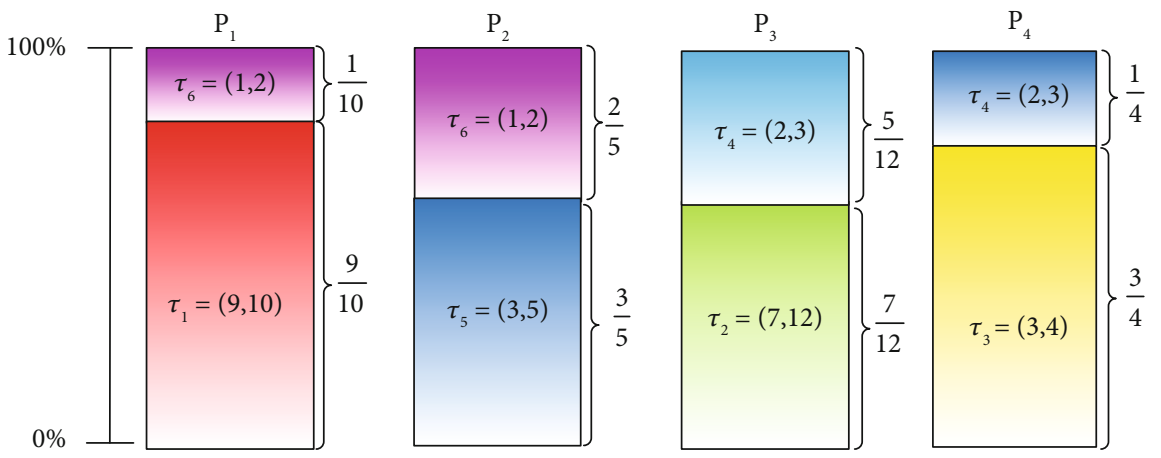

FIGURE 5: EDF-adaptive offline assigning tasks in 4 processors.

Figure 2 is EDF-fm's online execution phase that extends the earliest-deadline-first (EDF) scheduling to executed and migrating tasks. Specifically, each migrating task executes on two processors, and for each processor, at most two specific migrating tasks may execute upon it. Because the migrating tasks are prior to the fixed tasks for EDF-fm, the jobs of $\tau_{1}$ cannot meet any jobs' deadline although it shares a $9 / 10$ fraction of the processor $P_{1}$ of which the remanent utilization is given the migrating task $\tau_{2}$. The jobs of $\tau_{1}$ and $\tau_{4}$ and $\tau_{6}$ miss the deadline, and their total tardiness is 44 time units. The migrating tasks are $\tau_{2}, \tau_{3}$, and $\tau_{5}$, and 9 time unit is unused. If the task's utilization restriction of EDF-fm is violated, migrating tasks may miss deadlines, which invalidates the tardiness analysis.

Figure 3 is the offline assignment phase of EDF-os. All the tasks' utilizations are assigned to the processors in decreasing order using a worst- $t$ decreasing scheme rather than an arbitrary ordering. This phase tries to assign as many tasks as possible to be fixed; hence, the number of fixed tasks is as same as that of the processors at least. The remaining tasks are allocated nonzero shares from a succession of processors until the sum of its shares equals its utilization. Because the remaining tasks' utilization is considered in decreasing order, each processor must contain at least one fixed task with its utilization that is at least that of any migrating task. It is possible that such a task receives a nonzero share on only one processor, in which it is a fixed task; otherwise, it is migrating. This procedure ensures that there are at most two migrating tasks with nonzero shares on any processors. However, a migrating task under EDF-os can have nonzero shares on more than two processors. We can see there are $\tau_{1}, \tau_{2}, \tau_{3}$, and $\tau_{4}$ four tasks' utilization in decreasing order as fixed tasks and $\tau_{5}$ has nonzero shares on three processors. $P_{3}$ contains one fixed task $\tau_{3}$ and two migrating tasks $\tau_{5}$ and $\tau_{6}$.

Figure 4 is EDF-os's online execution phase. As in EDF$\mathrm{fm}$, migrating tasks are statically prioritized over fixed ones on any processor and each job executes on only one processor and fixed tasks are prioritized against each other using EDF. But if a processor has two migrating tasks $\tau_{i}$ and $\tau_{i+1}$ 


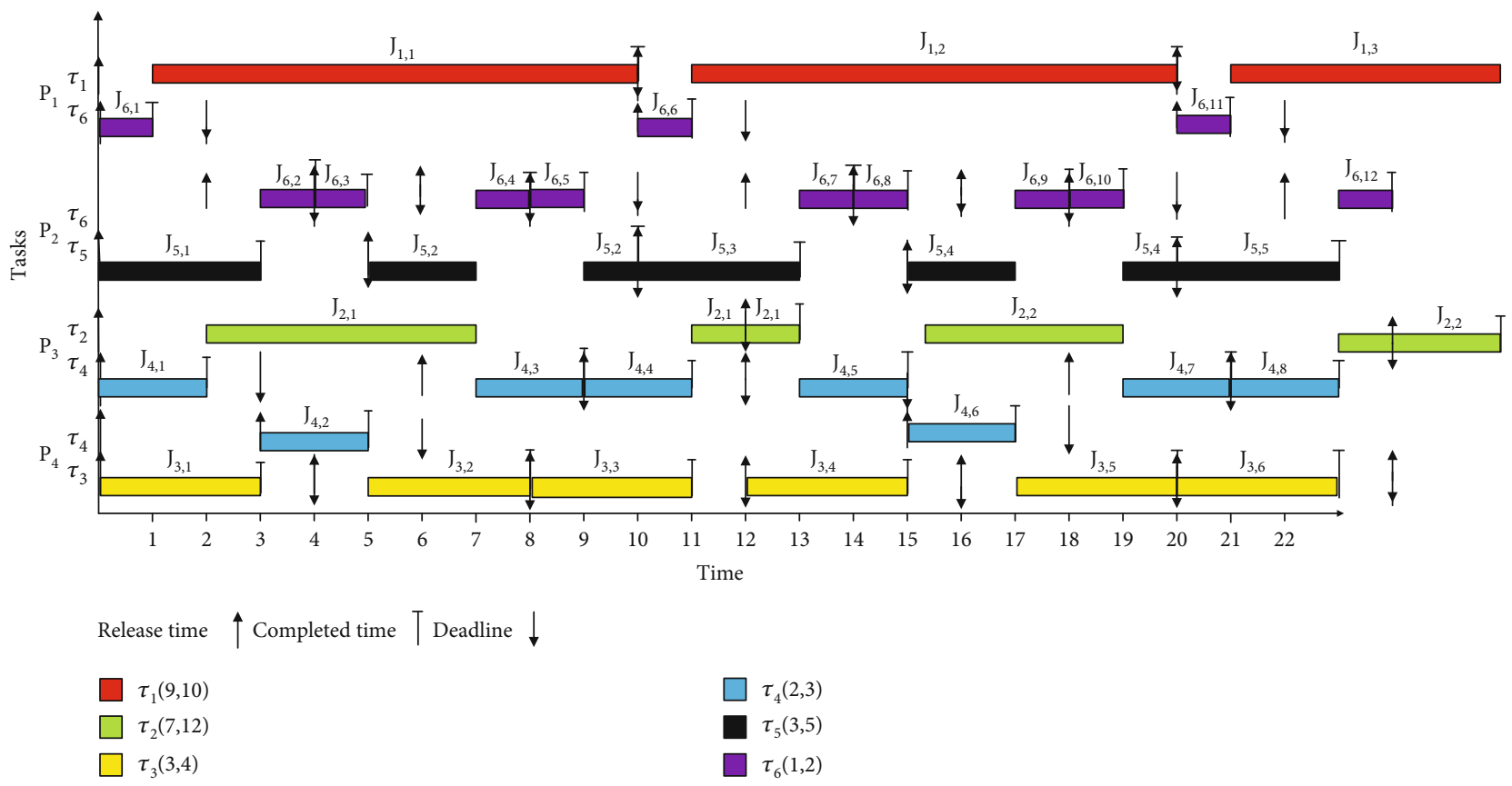

FIgURE 6: EDF-adaptive online scheduling results in 22 time units.

TABle 1: Statistics for the example in 22 time units.

\begin{tabular}{lcccc}
\hline Algorithm & Tardiness (time units) & The number of migrating tasks & Unused (time units) & Context switch (time units) \\
\hline EDF-fm & 44 & 3 & 9 & 15 \\
EDF-os & 15 & 3 & 1 & 29 \\
EDF-adaptive & 3 & 2 & 1 & 23 \\
\hline
\end{tabular}

assigned in this order, $\tau_{i}$ is statically prioritized over $\tau_{i+1}$. This means a migrating task executes with the highest priority on any processor that is not its first processor. Note that the lowest-indexed processor to which a migrating task $\tau_{i}$ is assigned jobs is called $\tau_{i}$ ' first processor. For example, $P_{2}$ is $\tau_{5}$ 's first processor and $P_{3}$ is $\tau_{5}$ 's second processor, so $\tau_{5}$ has the highest priority on $P_{3}$ and $\tau_{6}$ has higher priority than $\tau_{3}$.

EDF-os has better solutions for decreasing the number of jobs missing deadline, and it distributes the fixed tasks at first according to the processors, as shown in Figures 3 and 4 . It can be seen that $\tau_{2}$ and $\tau_{3}$ in the EDF-fm have been scheduled as migrating tasks and assign them as the fixed tasks in the EDF-os, whereas the task $\tau_{5}$ is split and assigned into three processors. The splitting will introduce more preemption overhead in the third processor. The jobs of $\tau_{1}$ and $\tau_{3}$ miss the deadline, and the total tardiness is 15 time units.

Figure 5 is the offline assignment phase of EDF-adaptive. Algorithm 1 divides these 4 (i.e., $K=4$ ) full utilization systems into 2 full utilization subsystems that are $\{(9,10),(3$, $5),(1,2)\}$ and $\{(9,10),(3,4),(2,3)\}$. In each subsystem, the sum of tasks' utilization is 2 . Meanwhile, the processors are divided into two parts $\left\{P_{1}, P_{2}\right\}$ and $\left\{P_{3}, P_{4}\right\}$ according to the subsystems, respectively. In each subsystems, as many tasks with the maximum period as possible are assigned as fixed tasks. We can see that the tasks with the minimum period such as $\tau_{6}$ and $\tau_{4}$ are the migrating tasks in the two subsystems, respectively.

Subsequently, according to Algorithm 2, we obtain the number of scheduling jobs for each task on processors. The scheduling results for this instance of EDF-adaptive are shown in Figure 6. We also consider the bounded tardiness restriction, i.e., the job is migrated at its boundary. Unlike EDF-os and EDF-fm, EDF-adaptive does not statically prioritized migrating tasks over fixed tasks. Instead, it always preempts the fixed tasks with the lowest utilization in its subsystems. It can be seen in Figure 6 that only the jobs of $\tau_{2}$ miss the deadline and the total tardiness is 3 time units. The migrating tasks are $\tau_{4}$ and $\tau_{6}$, and only 1 time unit is unused. The context switch overhead is lower than that in EDF-os. With the statistic results depicted in Table 1, we can find that EDF-adaptive is prior to EDF-fm and EDF-os at the total tardiness but the context switch times are between the two. These figures also show us that EDF-os and EDF-adaptive need to increase the migrating times, preemptive times, and context switch times to meet more jobs' deadlines.

\section{Evaluation}

In this section, we evaluate the performance of EDFadaptive by generating task sets for the SRT system and 


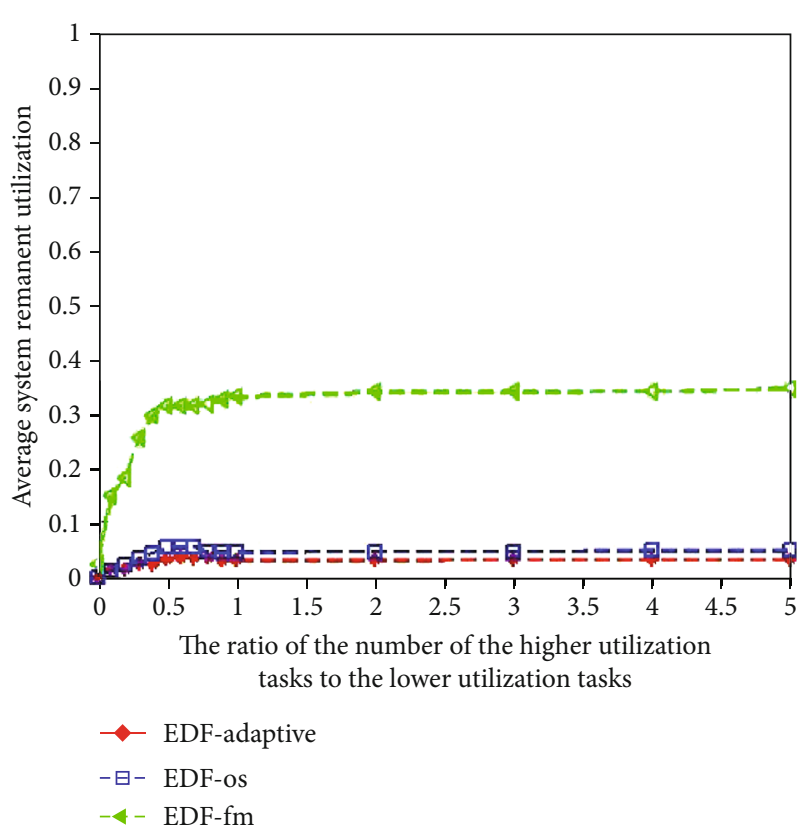

FIgURE 7: Average system remanent utilization.

compare it with EDF-fm and EDF-os against five criteria as follows:

(1) Average remanent utilization, which is the ratio of the sum of the unused time units of the processors to the total time units

(2) Average slack task minimum tardiness, according to Theorem 4

(3) Average context switching overhead, which is the multiplication of the times of context switching jobs and the overhead of per job context switching

(4) Migrating degree, which is the ratio of the number of the migrating tasks to the total number of tasks

(5) Splitting degree, which is the ratio of the number of the tasks split and scheduled on more than three processors to the total number of tasks

4.1. Task Set Generation. We used an Intel (R) core (TM) 2 Quad Q8400 multiprocessors workstation with 4 processors shared on $4 \mathrm{MB}$ L2 cache.

In the experiment, we randomly generated 100 task sets, and each task set has generated 20 soft real-time periodic tasks with different utilization and period parameters randomly. From each task set, three types of task sets, overloaded, underloaded, and all full utilization, are selected. We run each type of task set 10 times to calculate the average value. These tasks come with implicit deadlines and are either in $S^{H}$ or in $S^{L}$ because the utilization of half of them is less than 0.5 , and the other half is greater than or equal to 0.5 . The worst-case execution time (WCET) of each task is determined by the product of its period and utilization so that WCET of all tasks will be less than the deadline (which is equal to the period).

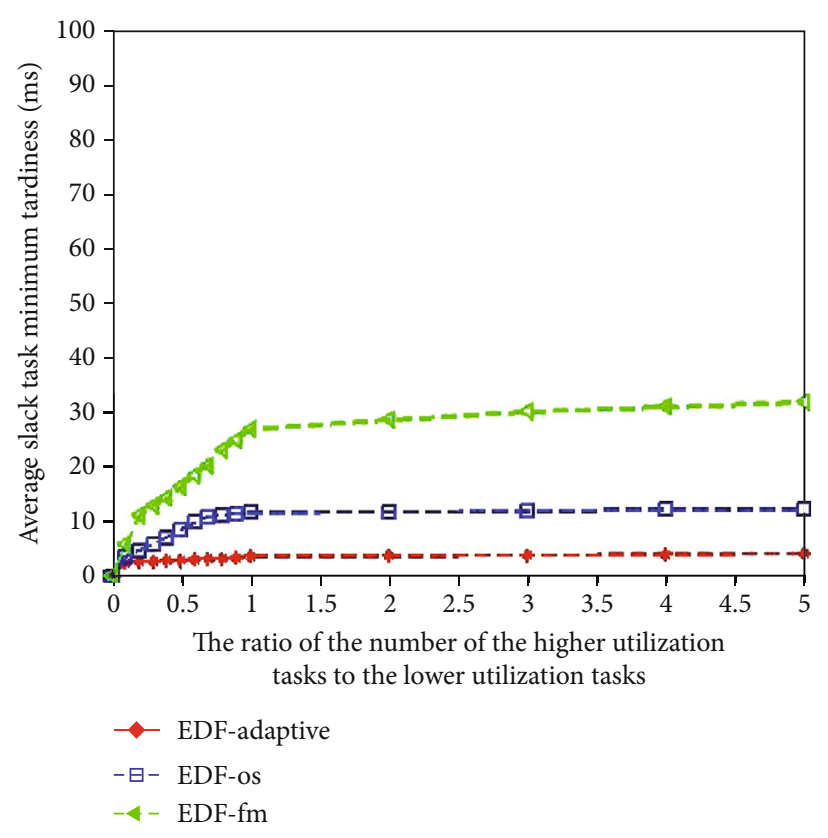

FIGURE 8: Average slack task minimum tardiness.

We set utilization caps in two sections, $[1,4]$ and $(4,5]$. This cap is a range when the sum of all task utilization is given. We calculate the system utilization and get the probable slack task according to Lemma 3. Then, we add or delete the probable slack task until an overloaded system, all full utilization system, or underloaded system is obtained. That is to say, when a set of 20 tasks is generated, the probable slack tasks will be added or subtracted so that the sum of the utilization of all tasks is less than 4 (underloaded system), equal to 4 (all full utilization system), or in the range of $(4,5]$ (overloaded system), respectively.

4.2. Results and Discussions. To evaluate the performance of EDF-adaptive, EDF-fm, and EDF-os, we ran 100 times and obtained the average of each evaluation criterion. The results are shown in Figures 7-11. In each figure, the horizontal axis is the ratio of the number of tasks in $S^{H}$ to the number of tasks in $S^{L}$, denoted as $R$. When the ratio increases, the system is moving from lower utilization to higher utilization.

Average remanent utilization. It can be seen from Figure 7 that for all algorithms, the remanent utilization increases with $R$, but it nearly stabilizes when $R$ is greater than 1. Overall, EDF-adaptive and EDF-os get smaller remanent utilization than EDF-fm. This is because EDF-fm selects the tasks and executes them in releasing sequence while EDF-os sorts the utilization of the tasks with descending order first before execution, and EDF-adaptive chooses tasks to execute according to their utilization.

Average minimum tardiness. Figure 8 shows that EDFadaptive and EDF-os have lower tardiness than EDF-fm because of their task selection, as it has been explained earlier. In fact, EDF-adaptive has the lowest tardiness because it can schedule the selective tasks that meet the full utilization system. 


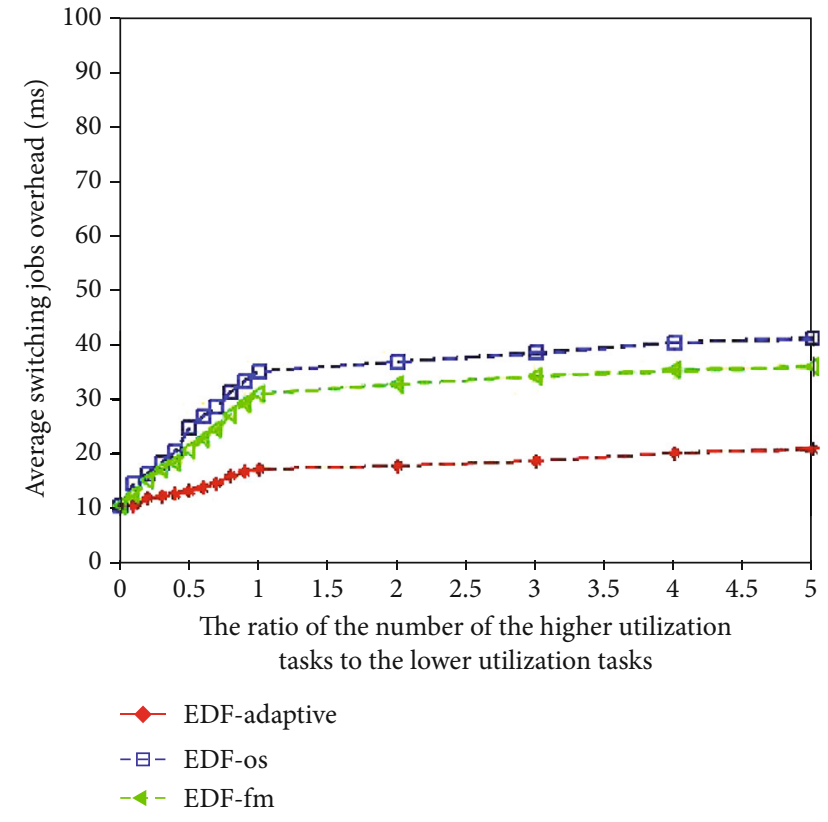

Figure 9: Average switching jobs overhead.

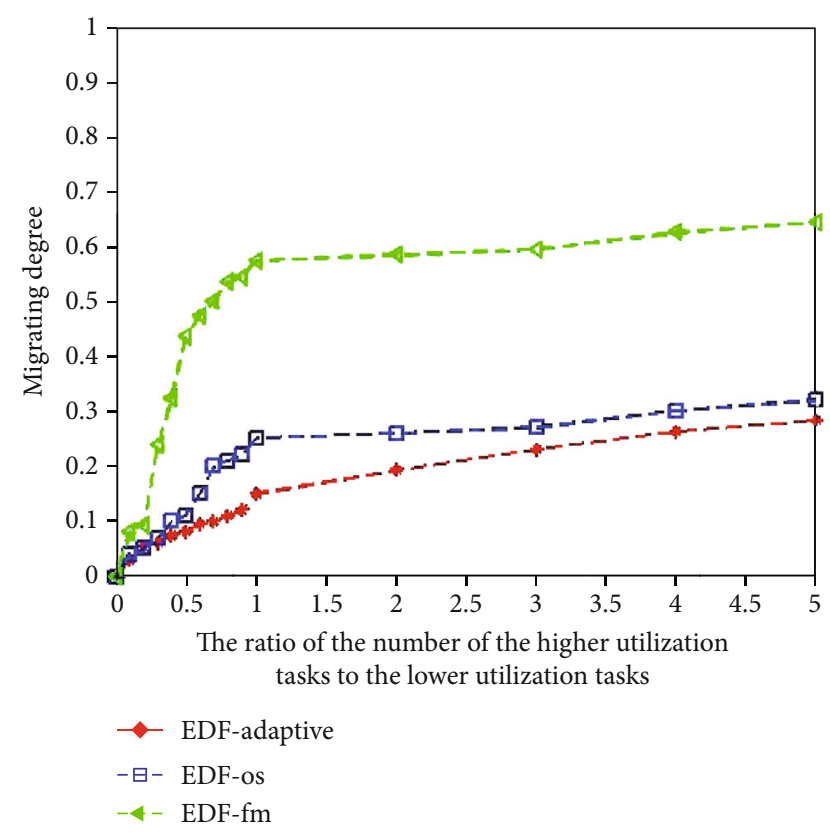

Figure 10: Migrating degree.

Average job switching overhead. Obviously, as shown in Figure 9, EDF-adaptive has lower overhead than EDF-os but has higher overhead than EDF-fm. Because EDF-adaptive and EDF-os are able to meet the jobs' deadlines, they have to increase the preemptive and migrating times which will give rise to context switch overhead. Although EDF-fm gets the lowest switch jobs overhead, many jobs miss their deadlines as shown in Figure 7. While EDF-os needs to meet the jobs' deadline and has no restrictions on task segmentation, some tasks may be partitioned into more than three processors that will introduce higher overhead than EDF-fm. EDFadaptive partitions tasks to meet $K$ full utilization rate in the

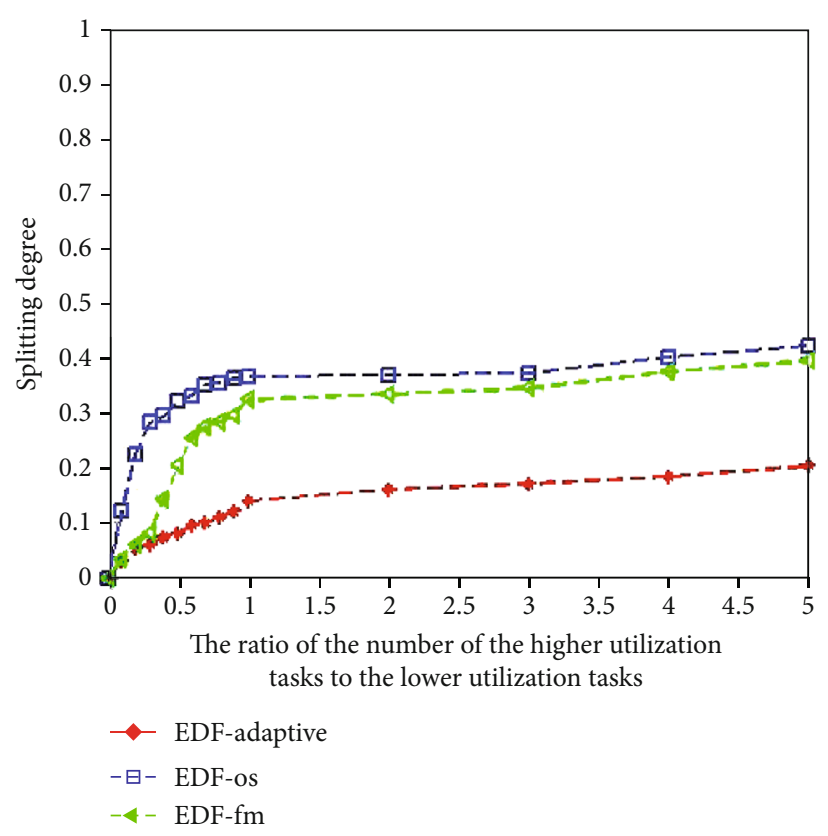

FIGURE 11: Splitting degree.

nondescending order integer value. As a result, it can reduce the switching cost of EDF-os. Obviously, EDF-adaptive can achieve almost as the same as EDF-os on system utilization and get the lowest tardiness in these algorithms and reduce the switch jobs overhead.

Migrating degree. Figure 10 shows that EDF-adaptive has a much smaller migration degree (thus lower migrating overhead) than EDF-fm and EDF-os, because there will be no migration between the different partition of processors when the maximal $K$ full utilization is achieved.

Splitting degree. The higher the splitting degree, the more tasks have been scheduled on multiple processors. It can be seen in Figure 11 that EDF-adaptive has performed much better than EDF-fm and EDF-os, in terms of preventing tasks from being split into multiple processors. Figure 11 also shows that EDF-os has the highest splitting degree. This is because EDF-os allows tasks to be assigned to more than 3 processors. This increases the partitioning of tasks. Based on the discussion above, we can conclude that EDF-adaptive is able to schedule more tasks in overloaded systems than EDF-fm and EDF-os. Because EDF-adaptive can selectively schedule from the task queue, it keeps the system running at full capacity as much as possible. For example, in an overloaded system, EDF-fm only schedules tasks in releasing sequence and EDF-os schedules tasks in descending order of utilization and then schedules migration tasks first, followed by fixed tasks. EDF-adaptive is to select appropriate tasks to schedule from $S^{H}$ and $S^{L}$. For example, EDF-os schedules tasks with a utilization of 0.3 in order, while EDF-adaptive can schedule two tasks with a utilization of, say, 0.1 and 0.2 . And in terms of job switching, task migration, and splitting, EDFadaptive complexity analysis. Despite better performance, EDF-adaptive suffers a higher time complexity than EDFfm and EDF-os. 
The time complexity of Algorithm 1 is $O\left(n^{2}\right)$ because the worst case for searching and selecting process is the total number of tasks $n$ in $S^{H}$ or $S^{L}$. And it uses a recursive approach for finding complementary tasks, mainly based on the number of $S^{H}$ or $S^{L}$. Algorithm 2 schedules tasks onto the processors, and the time complexity is $O(m)$, where $m$ is the number of processors. Hence, the time complexity of EDF-adaptive is $O\left(n^{2}+m\right)$, while EDF-fm has the time complexity $O(n m)$ and EDF-os has the time complexity $O(n+n m)$. In our future work, we will investigate approaches to reduce the time complexity and simplify the process. One idea is to use hash functions to find the complementary tasks from $S^{H}$ and $S^{L}$.

\section{Conclusions}

In this paper, we have proposed the EDF-adaptive algorithm for the soft real-time multiprocessor systems, which can be divided into three types of system: underloaded utilization system, full utilization system, and overloaded utilization system. The algorithm can allocate task sets onto the processors to achieve better performance than EDF-os and EDF$\mathrm{fm}$ in terms of the degree of task's splitting and migrating. However, partition problems are generally $\mathrm{Np}$ hard and EDF-adaptive is not optimal because of its time complexity. In the future, we will try to apply EDF-adaptive for the heterogeneous systems and mixed-criticality systems, while investigating new approaches to reduce the time complexity.

\section{Data Availability}

The data used to support the findings of this study are available from the corresponding author upon request.

\section{Conflicts of Interest}

The authors declare that they have no conflicts of interest.

\section{Acknowledgments}

The authors would like to thank Professor Feng Tian for critically reviewing the manuscript. The research and publication of this article were funded by the National Natural Science Foundation of China (No. 62171361), Scientific and Technological Project in Shaanxi Province (No. 2021 JM-440), and Industrial Science and Technology Research Project of Shaanxi Province of China (No. 2020GY-066).

\section{References}

[1] A. Bastoni, B. B. Brandenburg, and J. H. Anderson, "An empirical com-parison of global, partitioned, and clustered multiprocessor EDF schedulers," in Proc. 31st Int. Conf. IEEE Real-Time Systems Symposium, pp. 14-24, San Diego, CA, USA, 2010.

[2] H. S. Chwa, J. Lee, K. Phan, A. Easwaran, and I. Shin, "Global EDF schedulability analysis for parallel tasks on multi-core platforms," IEEE Transactions on Parallel and Distributed Systems, vol. 28, no. 5, pp. 1331-1345, 2017.
[3] L. George and J. F. Hermant, "A norm approach for the partitioned EDF scheduling of sporadic task systems," in Proc. 21st Euromicro Conf. Real-Time Systems, pp. 161-169, Dublin, Ireland, 2009.

[4] R. Ayari, I. Hafnaoui, and G. Beltrame, "ImGA: an improved genetic algorithm for partitioned scheduling on heterogeneous multi-core systems," Design Automation for Embedded Systems, vol. 22, no. 1-2, pp. 183-197, 2018.

[5] J.-M. Chung, S.-W. Jo, and M. H. Ahn, “ARQ supportive EDF scheduling for wireless networks servicing real-time applications," IEEE Communica-tions Letters, vol. 17, no. 7, pp. 1329-1331, 2013.

[6] J. Li, Z. Luo, D. Ferry, K. Agrawal, and L. Christopher Gill Chenyang, "Global EDF scheduling for parallel real-time tasks," Real-Time Syst, vol. 51, no. 4, pp. 395-439, 2015.

[7] G. Kedar, A. Mendelson, and I. Cidon, "SPACE: semipartitioned CachE for energy efficient hard real-time systems," IEEE Transactions on Computers, vol. 66, no. 4, pp. 717-730, 2017.

[8] E. M. Daoudiy and A. Kerfali, "Reducing the inter processor preemptions of the EDHS scheduling," in Proc. 5th Int.Conf. Optimization and Applications, pp. 1-6, Kenitra, Morocco, 2019.

[9] S. Moulik and Z. Das, "TASOR: a temperature-aware semipartitioned real-time scheduler," in Proc. IEEE Region 10 Conf. Technology, Knowledge, and Society, pp. 1578-1583, Kochi, IN-DIA, 2019.

[10] V. Bonifaci, G. D'Angelo, and A. M. Spaccamela, "Algorithms for hierarchical and semi-partitioned parallel scheduling," in Proc. 31st Int. Conf. IEEE Inter-national Parallel and Distributed Processing Symposium (IPDPS), pp. 738-747, Orlando, FL, 2017.

[11] A. Bastoni, B. B. Brandenburg, and J. H. Anderson, "Poster abstract: online semi- partitioned multiprocessor scheduling of soft real-time peri-odic tasks for QoS optimization," in Proc. 22nd Int. Conf. IEEE Real-Time and Embedded Technology and Appli-cations Symposium, pp. 1-1, Vienna, AUSTRALIA, 2016.

[12] J. Anderson, V. Bud, and U. Devi, “An EDF-based restrictedmigration scheduling algorithm for multiprocessor soft realtime systems," Real-Time Systems, vol. 38, no. 2, pp. 85-131, 2008.

[13] J. H. Anderson, J. P. Erickson, U M.-S. C. Devi, and B. N. Casses, "Optimal semi-partitioned scheduling in soft real-time systems," Journal of Signal Pro-cessing Systems, vol. 84, no. 1, pp. 3-23, 2016.

[14] S. Kato, Y. Ishikawa, and R. R. Rajkumar, "CPU scheduling and memory management for interactive real-time applications," Real-Time Systems, vol. 47, no. 5, pp. 454-488, 2011.

[15] J. P. Erickson, J. H. Anderson, and B. C. Ward, "Fair lateness scheduling: reducing maximum lateness in G-EDF-like scheduling," Real-Time Systems, vol. 50, no. 1, pp. 5-47, 2014.

[16] J. P. Erickson and J. H. Anderson, "Reducing tardiness under global scheduling by splitting jobs," in Proc. 25th Int. Conf. Real-Time Systems, ECE Grad Sch Engn, pp. 14-24, Paris, FRANCE, 2013.

[17] E. M. Daoudi, A. Dargham, A. Kerfali, and M. Khatiri, "Reducing the inter-processors migrations of the EKG algorithm," SCPE, vol. 19, no. 3, pp. 293-300, 2018.

[18] C. L. Liu and J. W. Layland, "Scheduling algorithms for multiprogramming in a hard-real-time environment," Journal of the 
Association for Computing Machinery, vol. 20, no. 1, pp. 4661, 1973.

[19] Y. Liu, X. Zhang, H. Li, and D. Qian, "Allocating tasks in multi-core processor based parallel systems," in Proc. 3rd Int. Conf. Network and Parallel Computing-Workshops, pp. 748753, Dalian, China, 2007.

[20] S. Senobary and M. Naghibzadeh, "First-fit semi-partitioned scheduling based on rate monotonic algorithm," in Proc. 1st Int. Conf. Intelligent Computing, Communication and Devices, SOA Univ, vol. 309, pp. 173-181, Bhubaneswar, INDIA, 2015.

[21] S. K. Dhall and C. L. Liu, "On a real-time scheduling problem," Operations Research, vol. 26, no. 1, pp. 127-140, 1978.

[22] W. A. N. G. Tao and L. I. U. Da-Xin, "The performance evaluation of rate monotonic tasks assignment algorithms on multiprocessor," Computer Science of China, vol. 34, no. 1, pp. 272-276, 2007.

[23] H. Xu and A. Burns, "A semi-partitioned model for mixed criticality systems," Journal of Systems and Software, vol. 150, pp. 51-63, 2019.

[24] A. Magdich, Y. Hadj Kacem, M. Kerboeuf, A. Mahfoudhi, and M. Abid, "A design pattern-based approach for automatic choice of semi-partitioned and global scheduling algorithms," Information and Software Technology, vol. 97, no. 5, pp. 83-98, 2018.

[25] H. A. Hassan, S. A. Salem, A. M. Mostafa, and E. M. Saad, "Deterministic harmonic segment-based semi-partitioning scheduling on multi-core real-time systems," ACM Transactions on Embedded Computing Systems, vol. 15, no. 4, pp. 129, 2016.

[26] S. Z. Sheikh and M. A. Pasha, "Energy-efficient multicore scheduling for hard real-time systems: a survey," ACM Transactions on Embedded Computing Systems, vol. 17, no. 6, pp. 126, 2019. 